\title{
Nonradiative Decay Channels for a Structurally-Distorted, Mono- Strapped BODIPY Derivative
}

\author{
Dumitru Sirbu, Joshua K. G. Karlsson and Anthony Harriman* \\ Molecular Photonics Laboratory, School of Natural and Environmental Sciences, Bedson Building, Newcastle Uni- \\ versity, Newcastle upon Tyne, NE1 7RU, United Kingdom.
}

\begin{abstract}
A boron dipyrromethene (BODIPY) derivative has been synthesized whereby a phenoxyl ring attached at the 3-position is bound through the oxygen atom to the boron center. This compound is structurally distorted, with the molecular surface being curved, and undergoes further geometrical perturbation at the excited-singlet state level. Fluorescence is readily observed in solution at ambient temperature, with the quantum yield rising with increasing viscosity of the surrounding solvent. Dual-exponential decay kinetics are observed, corresponding to E-type delayed fluorescence. In solution, the emission yield falls with increasing temperature but the opposite situation is found for the same compound dispersed in an amorphous sugar. These results are considered in terms of two radiationless decay channels. A viscosity-dependent avenue allows the fluorophore to function as a conventional fluorescent rotor for tracking changes in local rheology. A temperature-dependent channel leads to trapping within a new conformation, which is weakly coupled to the ground state but is able to repopulate the emitting state on a relatively slow timescale. Analysis of the experimental data allows estimation of some of the key kinetic parameters as a function of temperature.
\end{abstract}

\section{INTRODUCTION}

The term "fluorescent rotor" refers to a fluorophore that undergoes an environmentally sensitive conformational exchange which permits the emission intensity to report on changes in the local conditions. ${ }^{1-4} \mathrm{~A}$ popular fluorescent sensor is one where changes in rheology, most notably viscosity, can be assessed simply by monitoring the emission quantum yield. Many such molecular systems have been described in recent years, ${ }^{5-8}$ including those based on the well-known boron dipyrromethene (BODIPY) fluorophore. ${ }^{7,8}$ In this latter case, the normal conformational vector involves gyration of a meso-aryl ring around an unconstrained dipyrrin backbone. ${ }^{8}$ One way to rationalize the observed behavior is to invoke the concept that steric clashes between adjacent hydrogen atoms cause rupture of the potential energy surface for the excited-singlet state, introducing pinholes ${ }^{9}$ which facilitate rapid return to the ground state. Frictional forces between the rotating aryl ring and the surrounding medium serve to modulate the ease of gyration and provide the means by which to couple the emission quantum yield to changes in local viscosity. At a microscopic level, rotation of the meso-aryl ring causes slight buckling of the upper rim of the dipyrrin unit ${ }^{8}$ and it is this structural modification that underpins the sensory behavior. Alkyl substituents attached to appropriate sites on either the rotor or the dipyrrin upper rim dampen these structural oscillations and minimize the rotor effect. ${ }^{10}$

Surprisingly, attaching aryl groups to the lower rim of the dipyrrin unit also modulates the sensory properties but in a most unexpected manner." Thus, the 3,5-diarylBODIPY derivative is a poor rheology probe but the corresponding mono-functionalized 3-aryl derivative is the most effective BODIPY-based rotor identified to date! This finding poses a challenge to the conventional appreciation of the mechanics of the rotary behavior. In attempting to improve understanding of this situation, we note recent work $^{12}$ has established that the 3-aryl ring can be fixed in place by attaching it to the boron center via a C-O-B strap. In so doing, a new radiationless decay channel is opened that leads to delayed fluorescence from the BODIPY chromophore. The present report explores this deactivation process by recording the emission properties as a function of the fluidity of the surrounding medium. It is found that, relative to the corresponding rotors lacking the strap, ${ }^{11}$ the photophysical properties are complicated by competing structural changes. These findings might be useful in terms of designing highly specific fluorescent rotors for monitoring environmental change.

\section{RESULTS and DISCUSSION}

\section{Structural Details}

The target compound, BDFO (Figure 1), was synthesized $^{12}$ and purified as reported previously. The computed (B3 $\left.\mathrm{LYP} / 6-311 \mathrm{G}(\mathrm{d}) / \mathrm{PCM} / \mathrm{CHCl}_{3}\right)$ geometry indicates that the dipyrrin unit is severely distorted, in good agreement ${ }^{12}$ with the X-ray crystal structure (Supporting Information). In particular, the dipyrrin unit is curved and has the general appearance of a hemispherical dome since curvature is clearly apparent for both the dipyrrin and the strapped aryl ring (Figure 1). Distortion is also evident around the boron 

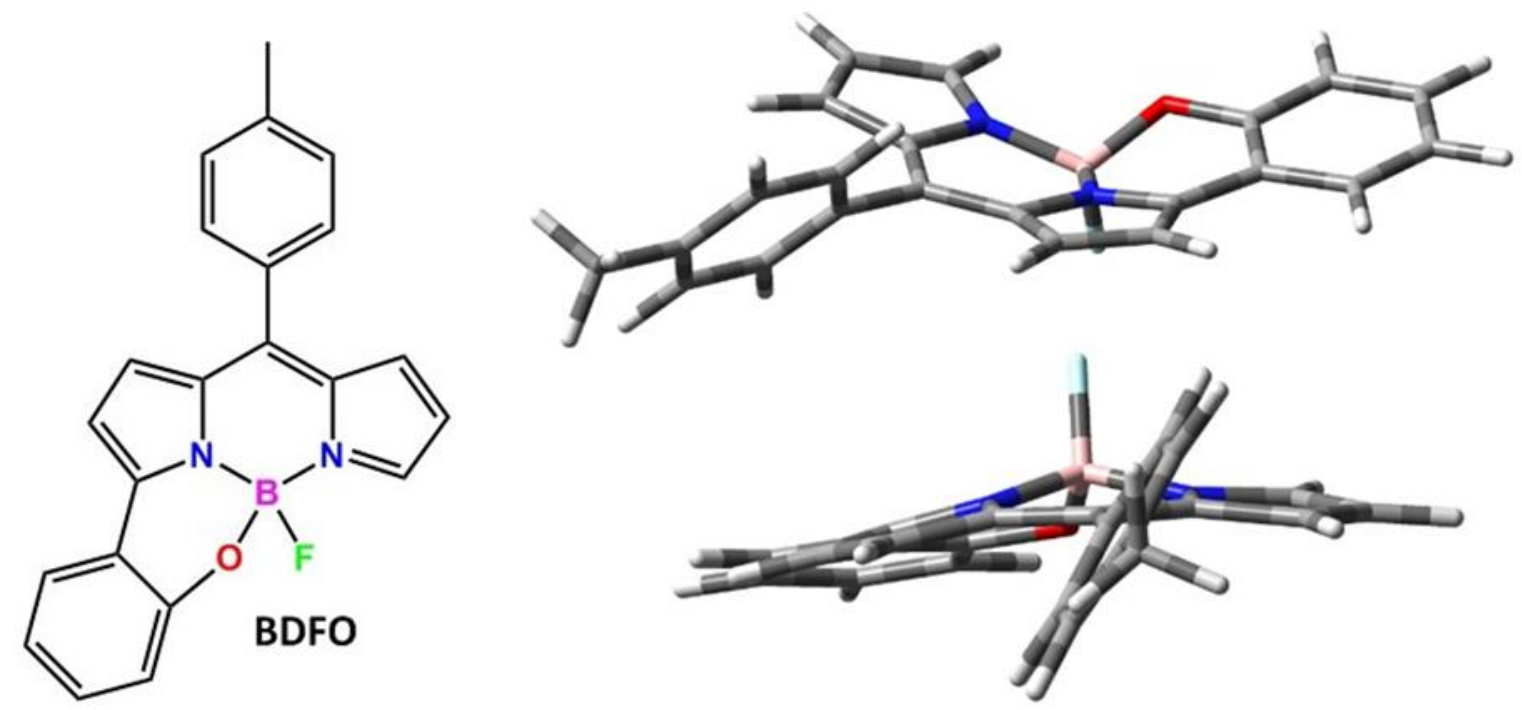

Figure 1. Chemical formula for the target compound, BDFO, and examples of the computed (B3LYP/6-311G(d)/PCM/CHCl $\left.{ }_{3}\right)$ energy-minimized geometry.

center, with N-B-N (N-B = $1.535 \AA$ and $1.539 \AA)$ and O-B-F

$\left(\mathrm{B}-\mathrm{O}=1.493 \AA\right.$ and $\mathrm{B}-\mathrm{F}=1.386 \AA$ ) angles of $108.2^{\circ}$ and $102.5^{\circ}$, respectively. The meso-aryl ring is held at an average dihedral angle of $56^{\circ}$ to the plane of the dipyrrin unit, taking into account that the latter is asymmetric because of the strap, but can oscillate around the mean position. The dipyrrin unit is not planar, as can be illustrated by the angle of $6.1^{\circ}$ along the $\mathrm{B}-\mathrm{C}$ (meso)-C(aryl) vector (Figure 1 ).

There is a small barrier of almost $1 \mathrm{kcal} / \mathrm{mol}$ for positioning the meso-aryl ring orthogonal to the dipyrrin unit (Figure 2a). Full rotation about the connecting $\mathrm{C}-\mathrm{C}\left(\mathrm{C}_{5}-\mathrm{C}_{6}-\mathrm{C}_{13}-\right.$ $\mathrm{C}_{14}$ ) bond requires concerted motion of the meso-substituent away from the dipyrrin and further bending of the pyrrole unit lacking the strapped aryl ring. This internal displacement provides a passage for rotation without contact between neighboring hydrogen atoms (see Supporting Information for details and examples of structures). The apparent energy barrier for full rotation of the meso-aryl ring $\left(\mathrm{C}_{5}-\mathrm{C}_{6}-\mathrm{C}_{13}-\mathrm{C}_{14}\right)$ is ca. $7 \mathrm{kcal} / \mathrm{mol}$ (Figure $\left.2 \mathrm{a}\right)$. Because of the collective distortions involving much of the molecular backbone, including atoms quite remote from the mesoposition, the rotational energy profile is relatively broad. The dihedral angle for the meso-aryl ring is likely to fluctuate around the mean position, imposing structural perturbations over the rest of the molecule, with only occasional passage over the larger barrier in solution at ambient temperature. As such, the ground-state landscape is expected to comprise a wide distribution of geometries at any given instance.

At the excited-singlet state level (TD-B3LYP/6-311G(d)/ $\left.\operatorname{PCM}\left(\mathrm{CHCl}_{3}\right)\right)$, the dipyrrin backbone is also distorted from planarity. The energy-minimized geometry (Figure S18) shows pronounced curvature for the dipyrrin unit, with $\mathrm{N}$ $\mathrm{B}-\mathrm{N}(\mathrm{N}-\mathrm{B}=1.523 \AA$ and $1.551 \AA)$ and $\mathrm{O}-\mathrm{B}-\mathrm{F}(\mathrm{B}-\mathrm{O}=1.509 \AA$
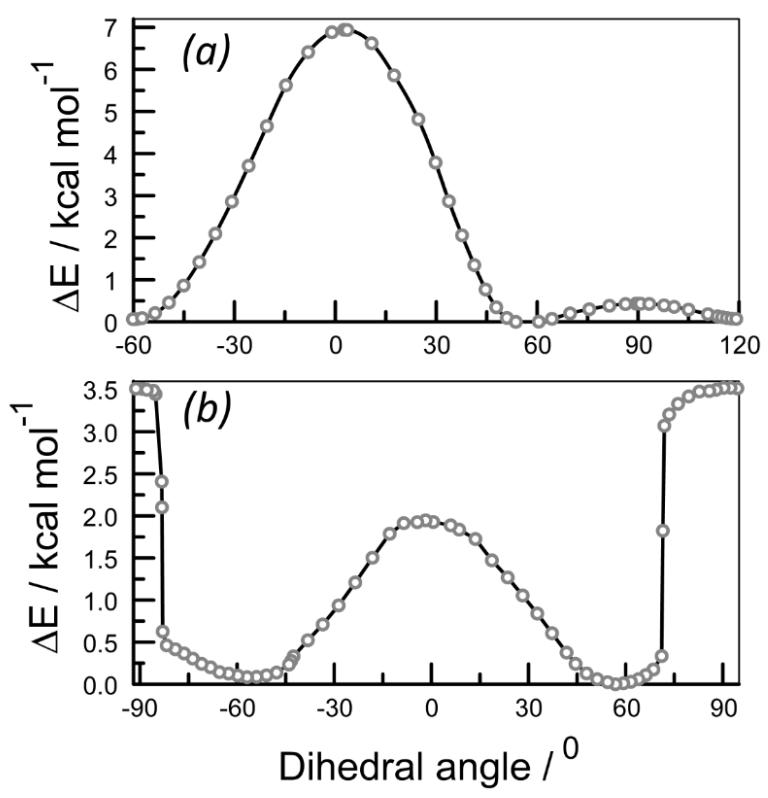

Figure 2. Plots showing the effect of dihedral angle for the meso-aryl ring $\left(\mathrm{C}_{5}-\mathrm{C}_{6}-\mathrm{C}_{13}-\mathrm{C}_{14}\right)$ on the total energy of the molecule as determined by the chain method (see Supporting Information for details) for (a) the ground state and (b) the firstexcited singlet state in a solvent reservoir.

and B-F $=1.380 \AA$ ) angles of $107 \cdot 7^{\circ}$ and $101.5^{\circ}$, respectively. The $\mathrm{C}_{5}-\mathrm{C}_{6}-\mathrm{C}_{13}-\mathrm{C}_{14}$ dihedral angle is calculated to be $57^{\circ}$ while the angle subtended by the $\mathrm{B}-\mathrm{C}($ meso)- $\mathrm{C}$ (aryl) axis is $11.4^{\circ}$. This latter angle, taken as a crude representative of structural distortion across the dipyrrin unit, exceeds that found for the ground state. Again, full rotation of the mesoaryl ring requires collective motions of the ring and the non-strapped pyrrole unit but the energy barrier $\left(E_{B}=2\right.$ $\mathrm{kcal} / \mathrm{mol}$ ) is significantly smaller than for the ground state (Figure $2 \mathrm{~b}$ ) - see the Supporting Information for a listing of the coordinates. Again, we might expect that the meso- 
aryl ring will oscillate around its mean position, with random passage over the modest barrier, giving rise to a wide variety of geometries.

These calculations are consistent with BDFO functioning as a molecular rotor ${ }^{1-4}$ where friction provided by the surrounding solvent layer will impede gyration of the meso-aryl ring. Three internal structural changes are implicated in the overall process: these involve (i) displacement of the rotor out of the plane defined by the meso- $\mathrm{C}$ and $\mathrm{B}$ atoms, (ii) bending of the pyrrole unit away from the meso-aryl ring so as to minimize steric clashes, and (iii) oscillation of the meso-aryl ring (see Supporting Information for further details). Each structural change will encounter resistance from neighboring solvent molecules and is likely to be inhibited in the solid state. Key geometry differences (Figure 2) between energy-minimized ground- and excited-states mean that there could be a significant Stokes' shift. It is also clear that there will be a wide variety of interconverting conformers in solution at ambient temperature at both ground- and excited-state levels.

\section{Photophysical and Electronic Properties}

In 2-methyltetrahydrofuran (MTHF) solution, BDFO ${ }^{12}$ exhibits a pronounced absorption maximum at $561 \mathrm{~nm}$ and a weak fluorescence maximum at $587 \mathrm{~nm}$ (Figures $\mathrm{S}_{1}$ and S2). The corresponding Stokes' shift is $790 \mathrm{~cm}^{-1}$, which is quite large for a BODIPY-based dye,13 while the emission quantum yield $\left(\Phi_{\mathrm{F}}\right)$ is determined to be $0.11 \pm 0.01$ at room temperature. Relative to conventional (i.e., non-rotor) BODIPY derivatives $\left(0.6<\Phi_{\mathrm{F}}<1.0\right),{ }^{14-16}$ the emission yield is low but, in comparison to the non-strapped, 3-aryl analogue $\left(\Phi_{\mathrm{F}}=0.013 \pm 0.002\right),{ }^{11}$ the fluorescence yield is unexpectedly high. This situation could be rationalized in terms of the rotor effect where $\Phi_{\mathrm{F}}$ is set by the ease of gyration of the meso-aryl ring. However, time-resolved emission studies indicate that, whereas fluorescence from the nonstrapped derivative decays via first-order kinetics with a lifetime $\left(\tau_{\mathrm{S}}\right)$ of $92 \pm 8 \mathrm{ps}$, emission from BDFO decays by way of a dual-exponential process according to Equation 1. In MTHF at $20{ }^{\circ} \mathrm{C}$, the two derived lifetimes are $\tau_{1}=350 \pm$ 25 ps $\left(\mathrm{A}_{1}=84 \%\right)$ and $\tau_{2}=1.4 \pm 0.1 \mathrm{~ns}\left(\mathrm{~A}_{2}=16 \%\right)$. Similar results, which remain independent of emission wavelength, were obtained under varying experimental conditions, including a few different excitation wavelengths, and for disparate samples of BDFO.

$$
I_{F}(t)=A_{1} e^{-\frac{t}{\tau_{1}}}+A_{2} e^{-\frac{t}{\tau_{2}}}
$$

Cyclic voltammetry carried out in deoxygenated $\mathrm{CH}_{2} \mathrm{Cl}_{2}$ containing background electrolyte indicates a quasi-reversible one-electron reduction step, ${ }^{17}$ with a half-wave potential of -1.11 V vs $\mathrm{Ag} / \mathrm{Ag}^{+}$and a peak separation of $75 \mathrm{mV}$ at a scan rate of $60 \mathrm{mV} / \mathrm{s}$. On oxidative scans, an irreversible wave is observed at a peak potential of $1.03 \mathrm{~V} \mathrm{vs} \mathrm{Ag} / \mathrm{Ag}^{+}$. This latter process appears to be a two-electron electrochemical step. To help assign the electrochemical results, a series of DFT calculations was performed in order to identify the main frontier molecular orbitals. Now, the LUMO appears to be closely associated with the BODIPY core with only a small amount of electron density off- loaded to either the meso-aryl ring, the strapped aryl ring or the bridging oxygen atom (Figure 3). In contrast, the HOMO involves both the dipyrrin unit and the strapped aryl ring, including the oxygen atom. This extended molecular orbital helps to reduce the band gap, thereby pushing the absorption maximum to lower energy, while the decreased overlap and increased spatial separation of HOMO and LUMO are likely to lower the excited singlet-triplet energy gap..$^{18,19}$ At the $\operatorname{HOMO}(-1)$ level, the electron density distribution moves from the dipyrrin unit to the strapped aryl ring, with a minor contribution from the unstrapped pyrrole unit (Figure 3). The meso-aryl ring does not contribute to either HOMO or $\mathrm{HOMO}(-1)$.
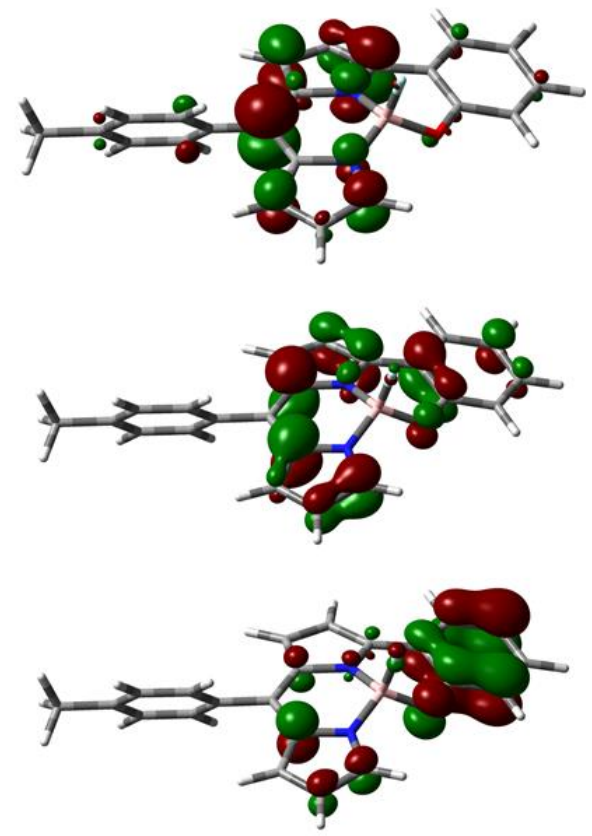

Figure 3. Kohn-Sham electronic isodensity plots of the LUMO (upper panel), HOMO (center panel) and HOMO(-1) (lower panel) for BDFO as computed at the DFT$6311(\mathrm{G}) \mathrm{d} / \mathrm{PCM} / \mathrm{CHCl}_{3}$ level.

With this information, electrochemical reduction appears to be fully consistent with formation of the dipyrrinbased $\pi$-radical anion. ${ }^{17}$ The first oxidation process is assigned to removal of an electron from the HOMO, the electrochemical band gap of $2.14 \mathrm{eV}$ (i.e., $580 \mathrm{~nm}$ ) being close to the HOMO-LUMO energy gap of $2.18 \mathrm{eV}$ (i.e., $570 \mathrm{~nm}$ ). These values can be compared with the optical band gap of $2.21 \mathrm{eV}$ (i.e., $561 \mathrm{~nm}$ ) calculated for the energy-minimized geometry. The second oxidative process is possibly related with the need to relieve steric strain imposed at the $\pi$-dication level, which is most easily achieved by breaking the $\mathrm{B}-\mathrm{O}$ bond and subsequent structural rearrangement. There is no obvious indication for the involvement of an intramolecular charge-transfer state (vide infra).

According to our computational studies, rotation around the connection between the dipyrrin unit and the mesoaryl ring has little effect on the energy of either the HOMO or $\mathrm{HOMO}(-1)$ (Figures S28 and S29). There is more effect 
Table 1. Summary of the spectroscopic properties derived by Gaussian analysis of the spectroscopic and photophysical properties recorded for BDFO in n-alkanols at $20^{\circ} \mathrm{C}$.

\begin{tabular}{|c|c|c|c|c|c|}
\hline Property & $\mathrm{CH}_{3} \mathrm{OH}$ & $\mathrm{C}_{2} \mathrm{H}_{5} \mathrm{OH}$ & $\mathrm{C}_{4} \mathrm{H}_{9} \mathrm{OH}$ & $\mathrm{C}_{7} \mathrm{H}_{15} \mathrm{OH}$ & $\mathrm{C}_{10} \mathrm{H}_{21} \mathrm{OH}$ \\
\hline$\eta / \mathrm{cP}$ & 0.594 & 1.095 & 2.54 & $5 \cdot 97$ & 11.50 \\
\hline$\lambda_{\mathrm{ABS}} / \mathrm{nm}$ & 559 & 559 & 560 & 560 & 561 \\
\hline$\lambda_{\mathrm{FLU}} / \mathrm{nm}$ & 580 & 582 & 584 & 586 & 587 \\
\hline$\Phi_{\mathrm{F}}$ & 0.13 & 0.16 & 0.20 & 0.25 & 0.27 \\
\hline$\tau_{1}\left(\mathrm{~A}_{1}\right) / \mathrm{ps}$ & 75 (0.95) & $110(0.92)$ & $140(0.83)$ & $170(0.79)$ & $230(0.65)$ \\
\hline$\tau_{2}\left(\mathrm{~A}_{2}\right) / \mathrm{ns}$ & 0.87 (0.05) & $0.91(0.08)$ & $1.09(0.17)$ & $1.08(0.21)$ & $1.25(0.35)$ \\
\hline$v_{\mathrm{oo}} / \mathrm{cm}^{-1}(\mathrm{a})$ & 17,275 & 17,210 & 17,175 & 17,140 & 17,135 \\
\hline FWHM / $\mathrm{cm}^{-1}(\mathrm{~b})$ & 915 & 870 & 865 & 855 & 830 \\
\hline $\mathrm{k}_{\mathrm{NR}} / 10^{8} \mathrm{~s}^{-1}$ & 13.0 & 10.7 & 8.3 & 6.4 & 5.1 \\
\hline
\end{tabular}

(a) Energy of the o,o transition derived from Gaussian fitting of the reduced emission band. (b) Full width at half-maximum of the individual Gaussian components used to indicate the relative broadness of the spectral band.

on the energy of the LUMO, especially as the two units become co-planar. In reality, however, the ground state is not expected to sample the structural regime where the HOMO-LUMO energy gap deviates far from the value computed for the energy-minimized geometry (Figure S29).

\section{Viscosity Effect}

In order to assess the significance of solvent viscosity on the emission properties of BDFO, a series of measurements was made in linear alcohol solvents at $20^{\circ} \mathrm{C}$. The nature of the solvent had a minor effect on the absorption $\left(\lambda_{\mathrm{MAX}}\right)$ and fluorescence $\left(\lambda_{\text {FLU }}\right)$ maxima, leading to a slightly enlarged Stokes' shift as the viscosity decreases. There was, however, a more substantial perturbation of the fluorescence quantum yield, which became amplified with increasing solvent viscosity $(\eta)$. Indeed, $\Phi_{\mathrm{F}}$ swelled from 0.16 in ethanol to 0.27 in decan-1-ol. It was noticeable that the fluorescence spectra were broadened in the shorter alcohol solvents. This effect (Figures S6-S1o) could be quantified by deconstructing the entire emission envelope into a set of five Gaussian components of common half-width (FWHM). On this basis, we see that the half-width falls steadily with increasing solvent viscosity (Table 1). Excitation spectra recorded under the same conditions do not show the same level of broadening as seen for emission (Figures $\mathrm{S}_{4}$ and $\mathrm{S}_{5}$, Table S2). The conclusion drawn from this work is that the excited-singlet state samples a wider conformational landscape than does the ground state and that the width of the distribution is dependent on solvent viscosity.

Across the series of linear alcohols, time-resolved emission profiles required analysis as the sum of two exponential components, according to Equation 1 (Figure 4). The derived lifetimes, $\tau_{1}$ and $\tau_{2}$, were sufficiently distinct for meaningful analysis ${ }^{20,21}$ and, in each case, consisted of a short-lived species with a lifetime around $0.2 \mathrm{~ns}$ and a longer-lived species having a lifetime around $1 \mathrm{~ns}$. The faster decaying component makes the major contribution toward the total signal in each solvent. The shorter lifetime, $\tau_{1}$, was found to increase progressively with increasing solvent viscosity whereas the longer lifetime, $\tau_{2}$, showed somewhat less sensitivity toward solvent viscosity. There was, however, a sharp rise in the contribution of the longer-lived component, $A_{2}$, with increasing viscosity. In fact, it is this higher fractional contribution of the second component that causes the quantum yield to increase at higher viscosity.

With conventional BODIPY-based rotors, ${ }^{6,7,11}$ time-resolved emission decay profiles could be well described in terms of single-exponential fits - leading to the conclusion that the longer lifetime is somehow related to the presence of the C-O-B strap. On making a crude approximation of the combined rate constant for nonradiative decay, $\mathrm{k}_{\mathrm{NR}}$, by Equation $2,{ }^{22}$ it is seen that the effect of viscosity is explained $^{23}$ reasonably well in terms of Equation 3 (Figure $\left.\mathrm{S}_{3}\right)$. Here, $v$ refers to the pressure exerted by the rotor as it twists through the medium and $\alpha$ is a coefficient that effectively relates to micro-viscosity. ${ }^{24}$ The activation barrier, $E_{A}$, can be considered to contain contributions from the structural change and from the temperature effect on the solvent viscosity. The value of $\alpha$ determined from a log-log plot is 0.44 (Figure $\mathrm{S}_{3}$ ), which is close to that found earlier for the unsubstituted BODIPY-based rotor $(\alpha=0.41)^{25}$ but significantly less than that found for the non-strapped 3aryl derivative $(\alpha=0.61) .{ }^{11}$ It might be noted that other expressions ${ }^{26,27}$ are available by which to correlate $\mathrm{k}_{\mathrm{NR}}$ with $\eta$, including the model developed by Kramers, ${ }^{28}$ which might be more suitable than the empirical formulation set by Equation 3. However, the scarcity of experimental data, together with the complexity of the system under study, precludes more elaborate analysis.

$$
\begin{aligned}
& A_{1}=\frac{k_{R A D}+k_{N R}-\left(1 / \tau_{2}\right)}{\left(\frac{1}{\tau_{1}}\right)-\left(\frac{1}{\tau_{2}}\right)} \\
& k_{N R}=\frac{v}{\eta^{\alpha}} e^{-\frac{E_{A}}{R T}}
\end{aligned}
$$




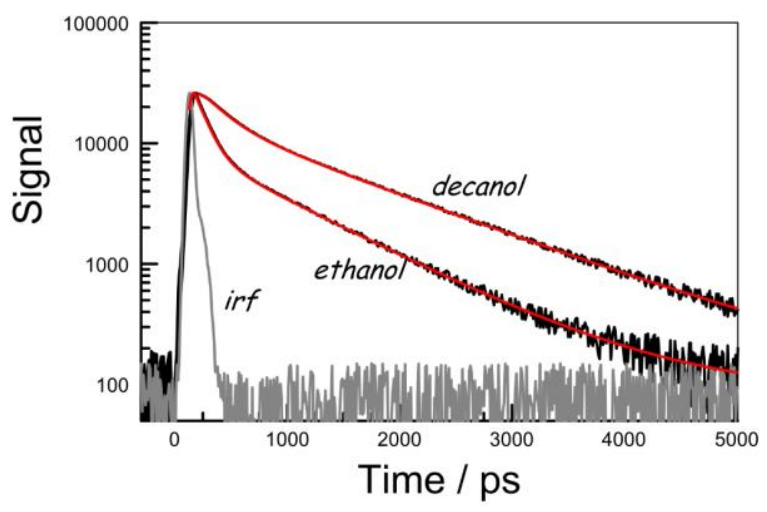

Figure 4. Examples of time-resolved fluorescence decay traces recorded for BDFO in alcohol solvents at room temperature. The excitation wavelength was $525 \mathrm{~nm}$. The instrument response function (irf) is shown in grey with the calculated fits appearing as red lines superimposed over the experimental data.

\section{Temperature Dependence in Fluid Solution}

Except for the dual-exponential decay profiles and viscosity-induced broadening of the fluorescence profiles, BDFO appears to function as a moderately effective molecular rotor. This belief was strengthened by the observation that $\Phi_{\mathrm{F}}$ increased steadily with decreasing temperature in MTHF (Figure 5). Indeed, in the rigid glass ${ }^{29}$ that forms at temperatures below ca. $100 \mathrm{~K}, \Phi_{\mathrm{F}}$ is close to unity. In the amorphous glassy region that persists between 100 and $140 \mathrm{~K}, \Phi_{\mathrm{F}}$ remains rather high but starts to decrease as the glass melts $(\mathrm{mp}=137 \mathrm{~K})$. The quantum yield continues to fall with increasing temperature and reaches a value of 0.11 at $20^{\circ} \mathrm{C}$. The profile for this temperature dependence cannot be explained in terms of the relaxation time of the solvent $^{30}$ or simply by changes in viscosity ${ }^{31}$ (Figure 5 ). Instead, the evolution of $\Phi_{\mathrm{F}}$ with temperature shows a more complex behavior, even when compared to related BODIPY-based molecular rotors in the same solvent.

The energy of the emission maximum is at its highest in the rigid glass, formed at temperatures below ca. $100 \mathrm{~K}$, and falls sharply as the solvent enters the amorphous glassy phase. The solvent melts at $137 \mathrm{~K}$, thereafter the emission maximum increases gently with rising temperature, although this effect is modest. These changes are possibly associated with variations in solvent polarizability or polarity (Figures $\mathrm{S}_{14}$ and $\mathrm{S}_{15}$, Tables $\mathrm{S}_{4}$ and $\mathrm{S}_{5}$ ). ${ }^{2}$

According to our Gaussian analysis, the band half-width exhibits a pronounced temperature dependence in fluid solution. In fact, the FWHM is small in the rigid glass but increases across the amorphous region. Once the solvent melts at $137 \mathrm{~K}$, the individual bands begin to broaden with increasing temperature. This latter effect is illustrated by the inset to the upper panel in Figure 5 and is emphasized in Figure 6. In fluid solution, the FWHM is significantly larger than found for conventional BODIPY-based dyes and is explained in terms of the molecule adopting an unusually wide distribution of geometries that interconvert slowly on the timescale for emission. This structural heterogeneity increases with increasing temperature and/or decreasing viscosity; the same effect is apparent for the linear alcohols at room temperature.
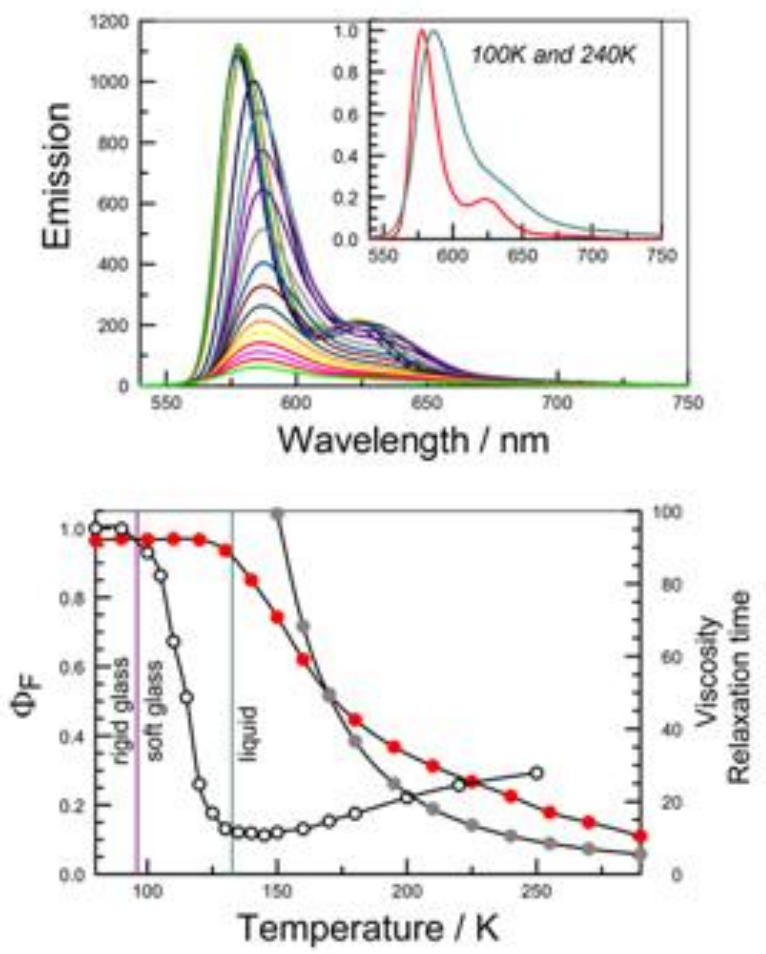

Figure 5. Fluorescence spectral evolution as a function of temperature for BDFO in MTHF (upper panel) and the effect of temperature on the fluorescence quantum yield (red points) for the same experiment (lower panel). The inset compares spectra recorded at low (red curve) and high (blue curve) temperatures. Also given on the lower panel are the temperature dependences for the solvent viscosity (in units of $\mathrm{cP}$, grey points) and the emission maximum (in units of $\mathrm{cm}^{-1}$ ) for Oxazine-4 in MTHF (a dye reported $3^{\circ}$ to respond exclusively to changes in solvent relaxation time) (open circles). These latter values have been normalized for convenient display and the actual values are given in Tables $\mathrm{S}_{4}$ and $\mathrm{S}_{5}$.

All indications suggest that this broadening is inhomogeneous in nature. ${ }^{33-36}$ Certainly, the spectral band-shape is well represented by Gaussian profiles but cannot be analyzed as Lorentzian (Figure S12). The broadening is not apparent in the rigid glass but increases progressively with increasing temperature in fluid solution, especially towards higher temperatures. A small increase in $v_{\mathrm{o}, \mathrm{o}}$ accompanies the spectral broadening (Table $\mathrm{S}_{3}$ ). The broadening does not depend on excitation wavelength but, at a given temperature, is restricted by increased viscosity. This points towards some type of structural perturbation as playing a key role. This geometry change could be related to nuclear displacements around the dye molecule and its interaction with the solvent. ${ }^{37,38}$ While the major contributor to the observed spectral broadening most likely stems from the distribution of molecular geometries at ground and excited state levels, ${ }^{39,40}$ it should be stressed that linear 
spectroscopy of the type applied here does not allow meaningful discrimination between homogeneous and inhomogeneous broadening.

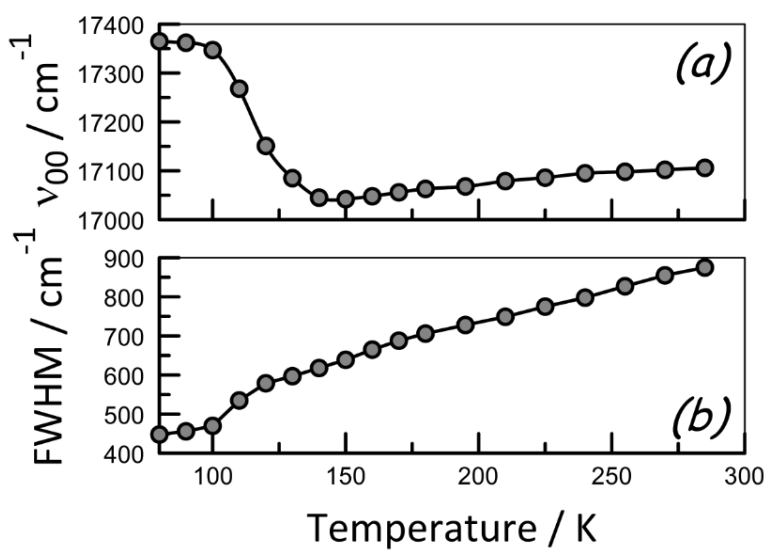

Figure 6. Effect of temperature on the fluorescence spectroscopic properties observed for BDFO in MTHF. Individual panels refer to (a) the emission maximum, (b) the band halfwidth for deconstructed Gaussian-shaped components.

\section{Temperature Dependence in the Glassy State}

In an effort to isolate structural changes from possible temperature effects, samples of BDFO were dispersed in sucrose octaacetate (SOA) and cast into a transparent block. At room temperature, very strong fluorescence was recorded with a quantum yield of $0.94 \pm 0.04$, as measured with an integrating sphere and excitation at $525 \mathrm{~nm}$. Timeresolved fluorescence studies indicated that the decay profiles for BDFO in SOA at room temperature could be analyzed satisfactorily in terms of a dual-exponential process with lifetimes of $0.35 \pm 0.05 \mathrm{~ns}\left(\mathrm{~A}_{1}=31 \%\right)$ and $9.1 \pm 0.3 \mathrm{~ns}$ $\left(A_{2}=69 \%\right)$. A fluorescence lifetime of $9 \mathrm{~ns}$ is too high for a BODIPY-based dye since the radiative lifetime is usually on the order of ca. 5 ns or less. ${ }^{41}$ It might be noted that Rhodamine 6G dispersed in SOA is strongly fluorescent $\left(\Phi_{\mathrm{F}}=0.95\right.$ \pm 0.4 ) and shows a single exponential decay profile with a lifetime of $4.3 \pm 0.1 \mathrm{~ns}$.

Fluorescence studies were carried out as a function of temperature for BDFO in the SOA matrix. The emission intensity increased progressively as the temperature was raised from 80 to $340 \mathrm{~K}$ (Figure 7 ). This behavior is in marked contrast to that reported above for MTHF solution. The emission maximum $\left(v_{\mathrm{oo}}=17,100 \mathrm{~cm}^{-1}\right)$ remained approximately constant across this temperature range. The corresponding band half-width, FWHM, increased slightly with temperature but this effect is nowhere near as pronounced as that observed in fluid MTHF.

For fluorophores embedded in a soft matrix, phonon interactions are to be expected and this situation will broaden the natural linewidth according to a temperature dependent dephasing term. The significance of this latter term will differ according to the environment surrounding the emitter. Dye molecules within the matrix will experience local perturbations due to the different locations, which can cause disparate electric fields. This field might disturb the electronic levels (i.e., the Stark effect) and thereby modify the transition energy. This is insignificant for BDFO in SOA. The overall effect, however, is that the spectral profile deviates from Lorentzian (Figure S12). In fact, the temperature dependence recorded for the band half-width follows a power law dependence, indicating that the inhomogeneous broadening arises from sampling different environments. ${ }^{42}$

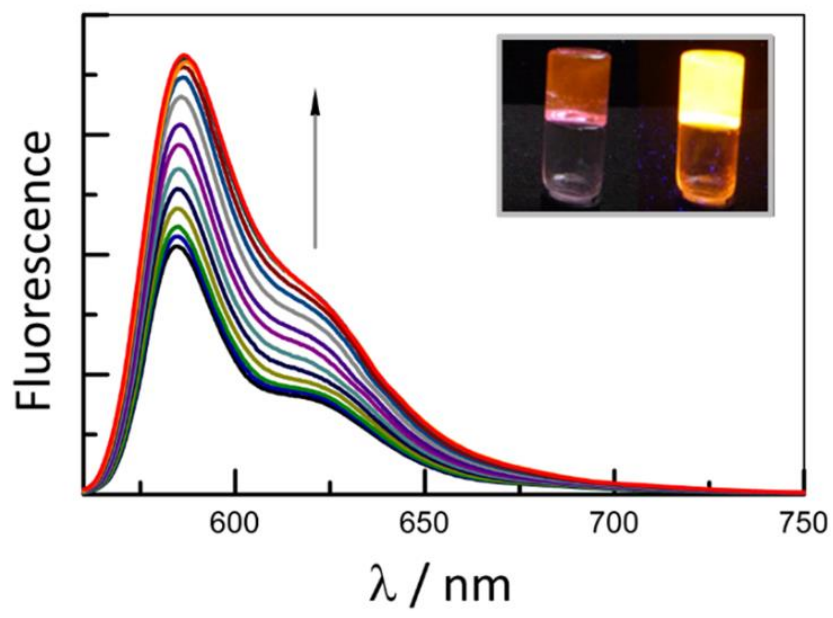

Figure 7. Effect of temperature on the fluorescence spectral profile recorded for BDFO in an amorphous SOA gel. The inset shows the sample (half-filling an inverted glass tube) in the dark and under UV excitation while the arrow indicates the direction of increasing temperature.

$$
\begin{aligned}
& \tilde{\mathrm{n}}=\frac{\Phi_{F}}{\Phi_{P F}}-1=\frac{\Phi_{T} \times \Phi_{S}}{1-\Phi_{S} \Phi_{T}} \\
& \Phi_{P F}=\frac{k_{R A D}}{k_{R A D}+k_{N R}}=k_{R A D} \times \tau_{F} \\
& \Phi_{T}=\frac{k_{N R}}{k_{R A D}+k_{N R}} \\
& \Phi_{S}=\frac{k_{D S} \exp ^{-\Delta E / R T}}{k_{D S} \exp ^{-\Delta E / R T}+k_{G}} \\
& \Phi_{F}=\Phi_{P F}\left(1+\Phi_{S} \Phi_{T}+\left(\Phi_{S} \Phi_{T}\right)^{2}+\cdots\right)
\end{aligned}
$$

The increasing $\Phi_{\mathrm{F}}$ with rising temperature found in glassy SOA is reminiscent of the delayed luminescence ${ }^{43}$ observed in certain conducting polymers, ${ }^{44,45}$ fullerenes ${ }^{46}$ and organic dyes. ${ }^{47,48}$ A similar effect was reported ${ }^{49}$ recently for a BODIPY dye equipped with a redox-active strap attached to the boron center. In fact, the temperature dependence can be fully explained by slight adaptation of the model for thermally-activated, delayed fluorescence developed by Baleizão and Berberan-Santos ${ }^{50}$ and by Dias et al..$^{1}$ In our version, part of the initial population of the emitting state, $S_{1}$, decays via prompt fluorescence with a rate constant of $\mathrm{k}_{\mathrm{RAD}}$. Nonradiative decay, occurring with a global rate constant of $\mathrm{k}_{\mathrm{NR}}$, accounts for decay of the remainder of the emitting state. This latter radiationless pathway leads to formation of a molecular conformation distinct from those of either the ground state or the emitting state. Deactivation of this new state can lead to repopulation of the emitting $S_{1}$ state, with a rate constant $k_{D S}$, or formation of the ground state, with a rate constant $\mathrm{k}_{\mathrm{G}} \cdot{ }^{52}$ 
Table 2. Compilation of the parameters relating to the temperature-dependent fluorescence quantum yield derived for BDFO in the SOA glass ${ }^{a}$ and in MTHF solution. ${ }^{b}$

\begin{tabular}{|l|l|l|l|l|l|l|l|}
\hline Medium & $\mathrm{k}_{\mathrm{RAD}} / 10^{7} \mathrm{~s}^{-1}$ & $\mathrm{k}_{\mathrm{DS}} / 10^{9} \mathrm{~s}^{-1}$ & $\mathrm{k}_{\mathrm{NR}} / 10^{7} \mathrm{~s}^{-1}$ & $\mathrm{k}_{\mathrm{G}} / 10^{7} \mathrm{~s}^{-1}$ & $\mathrm{k}_{\mathrm{PH}} / 10^{9} \mathrm{~s}^{-1}$ & $\Delta \mathrm{E} / \mathrm{kJ} \mathrm{mol}-1$ & $\mathrm{EP}_{\mathrm{P}} / \mathrm{kJ} \mathrm{mol}^{-1}$ \\
\hline SOA & 13 & 63 & 14.5 & 52 & $\mathrm{NA}$ & 7.5 & NA \\
\hline MTHF & 13 & 19 & 0.35 & 22 & 28 & 8.1 & 6.0 \\
\hline
\end{tabular}

(a) Values derived by iterative fitting across the full temperature range to Equations 4-10. (b) See supporting Information.

Table 3. Comparison of calculated and observed fluorescence lifetimes for BDFO in the SOA glass at selected temperatures.

\begin{tabular}{|l|l|l|l|l|l|l|}
\hline Temperature / K & $\Phi_{\mathrm{F}}$ & $\tilde{\mathrm{n}}$ & $\tau_{\mathrm{PF}} / \mathrm{ns}{ }^{(\mathrm{a})}$ & $\tau_{\mathrm{DF}} / \mathrm{ns}{ }^{(\mathrm{b})}$ & $\tau_{1}\left(\mathrm{~A}_{1}\right) / \mathrm{ns}^{(\mathrm{c})}$ & $\tau_{2}\left(\mathrm{~A}_{2}\right) / \mathrm{ns}(\mathrm{d})$ \\
\hline 150 & 0.475 & 0.06 & 2.4 & 22.6 & $2.60(94 \%)$ & $19.8(6 \%)$ \\
\hline 170 & 0.655 & 0.20 & 1.7 & 15.3 & $1.65(83 \%)$ & $14.7(17 \%)$ \\
\hline 210 & 0.825 & 0.8 & 0.90 & 10.8 & $1.00(55 \%)$ & $11.2(45 \%)$ \\
\hline 240 & 0.900 & 1.6 & 0.55 & 9.7 & $0.65(39 \%)$ & $10.1(61 \%)$ \\
\hline 290 & 0.910 & 2.2 & 0.33 & 9.0 & $0.35(31 \%)$ & $9.1(69 \%)$ \\
\hline
\end{tabular}

(a) Fast decaying component calculated from Equation 9. (b) Slow decaying component calculated from Equation 10. (c) Shorter lifetime (and its fractional contribution) observed in the time-resolved emission studies. (d) Longer lifetime (and its fractional contribution) observed experimentally.

To account for the temperature dependence noted for $\Phi_{\mathrm{F}}$, it is necessary to propose that $\mathrm{k}_{\mathrm{DS}}$ involves crossing a modest energy barrier, $\Delta \mathrm{E}$, and that $\mathrm{k}_{\mathrm{G}}$ is relatively slow. We can impose a few additional restrictions on the model that allow refinement of the numerical values for the various rate constants. These include the mean number (ñ) of cycles from the conformer back to $S_{1}$ (Equation 4 ) and definition of the quantum yields for prompt fluorescence $\left(\Phi_{\mathrm{PF}}\right)$ and for formation of the conformer $\left(\Phi_{\mathrm{T}}\right)$ in terms of $k_{\mathrm{RAD}}$ and $\mathrm{k}_{\mathrm{NR}}$ (Equations 5 and 6). The probability for repopulation of $S_{1}$ from the conformer $\left(\Phi_{S}\right)$ is expressed in Equation 7, where allowance is made for the thermally activated step. Now, the overall fluorescence quantum yield, $\Phi_{\mathrm{F}}$, at any temperature is given by Equation 8 . To aid analysis of the temperature dependent $\Phi_{\mathrm{F}}$ values, we have fixed $\mathrm{k}_{\mathrm{RAD}}$ at $1.3 \times 10^{8} \mathrm{~s}^{-1}$, as indicated by application of the Strickler-Berg expression, ${ }^{33}$ and we note that $\tilde{n}$ can be used to refine the product of $\Phi_{\mathrm{T}}$ and $\Phi_{\mathrm{S}}$ according to Equation 4 .

Within the model, ${ }^{00,51}$ the various rate constants can be used to estimate the lifetimes for prompt $\left(\tau_{\mathrm{PF}}\right)$ and delated $\left(\tau_{\mathrm{DF}}\right)$ fluorescence at any given temperature, according to Equations 9 and 10, where $\tau_{\mathrm{F}}$ is defined in Equation 5. These calculated values can be compared to experimental lifetimes measured at selected temperatures and used to further refine the rate constants extracted from the global fits. The final values are collected in Table 2 while the fit to the temperature dependent $\Phi_{\mathrm{F}}$ values is shown as Figure 8, together with an illustration of the model.

It can be seen that $\mathrm{k}_{\mathrm{G}}$, the rate constant for escape from the trap to the ground state, is low relative to the rate constant, $\mathrm{k}_{\mathrm{DS}}$, for reverse population of the emitting state. The energy barrier separating the trap from the emitting species is small, being around ${ }_{3} \mathrm{RT}$ at room temperature, and this emphasizes the importance of the delayed fluorescence pathway for the SOA glass. The derived parameters give a very good representation of the emission lifetimes
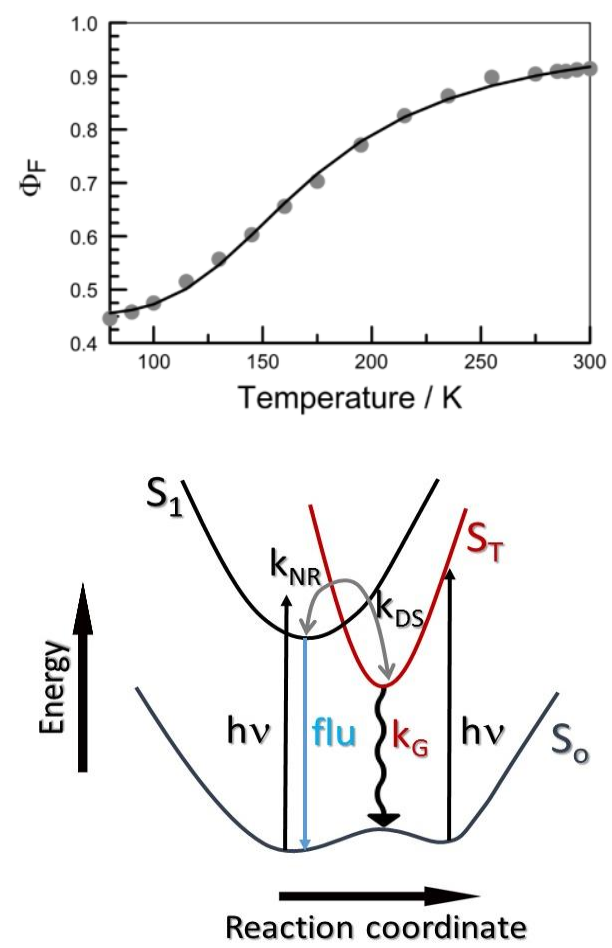

Figure 8. The upper panel shows the effect of temperature on the fluorescence quantum yield, the solid line refers to a fit to Equation 9. Shown below is a potential energy diagram intended to illustrate the model used to explain the temperature dependence in the soft SOA gel. Note the inclusion of direct absorption to the conformational trap. 
measured over the respective temperature range (Table 3 ). From the fractional amplitudes of the shorter- and longerlived components seen in the time-resolved emission decay curves at room temperature, we can estimate ${ }^{52}$ the

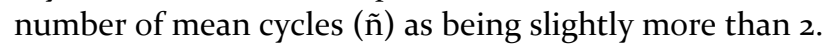

$$
\begin{gathered}
\tau_{P F}^{-1}=k_{R A D}+k_{N R}+k_{D S} \exp ^{-\Delta E / R T} \\
\tau_{D F}^{-1}=\frac{k_{G}+k_{D S} \exp ^{-\Delta E / R T}\left(1-\Phi_{T}\right)}{1+\tau_{F}\left(k_{D S} \exp ^{-\Delta E / R T}\right)}
\end{gathered}
$$

\section{Radiationless Decay in Fluid Solution}

Complete rotation of the meso-aryl ring will be curtailed in the SOA glass but, according to our model, certain structural changes are facilitated in this soft gel. Internal distortions are less likely in the rigid MTHF glass that forms below about $100 \mathrm{~K}$ and, as a consequence, $\Phi_{\mathrm{F}}$ approaches unity under such conditions. This situation contrasts with that for SOA where $\Phi_{\mathrm{F}}=0.38$ at $8 \mathrm{oK}$. We can rationalize this disparity on the basis that slow cooling of the sample in MTHF achieves the lowest-energy conformation at $80 \mathrm{~K}$ whereas the SOA sample is prepared by heating the mixture to $100{ }^{\circ} \mathrm{C}$ before cooling rapidly. This latter protocol is likely to create a distribution of higher energy geometries. Thus, the starting structures are different, with SOA favoring increased levels of structural distortion that do not relax to the energy-minimized geometry on solidification. For this reason, direct excitation to the trap has been included as a possibility on Figure 8 for SOA. In fluid solution, we anticipate that both gyration of the meso-aryl ring and distortion of the dipyrrin backbone become more favorable with increasing temperature. These internal motions are considered to be concerted, on the basis of the structural calculations reported earlier. To explain the situation expressed in Figure 5, it is necessary to impose the condition that rotation of the meso-aryl ring promotes fast nonradiative return of the emitting state to the ground state. 54

We had previously proposed that this rotation ruptures the potential energy surface of the $S_{1}$ state so as to cause the appearance of pinholes through which the exciton can escape. ${ }^{55,56}$ It is now possible to combine these two events into a model relevant to BDFO in fluid solution. Here, the situation illustrated in Figure 8 to account for BDFO in a soft glass is modified by the inclusion of a pinhole ${ }^{55}$ on the $\mathrm{S}_{1}$ potential surface that acts as an escape route for irreversible return to the ground state. This pinhole is accessed as the meso-aryl ring twists about the connecting axis (Figure 9). It is the equivalent of a conical intersection between the two potential energy surfaces but it might not be so well defined. It might be argued that several such pinholes might appear on the surface as the structural changes take place at the excited state level. Conical intersections might be construed as having a specific geometry so the term pinhole, intended to be much looser, appears more suitable for the present case.

The so-called pinhole (or conical intersection) could be associated with either (or both) the excited-state surface or with the conformational trap $\left(\mathrm{S}_{\mathrm{T}}\right)$. In fact, it proved impossible to obtain a meaningful fit to the quantum yields and

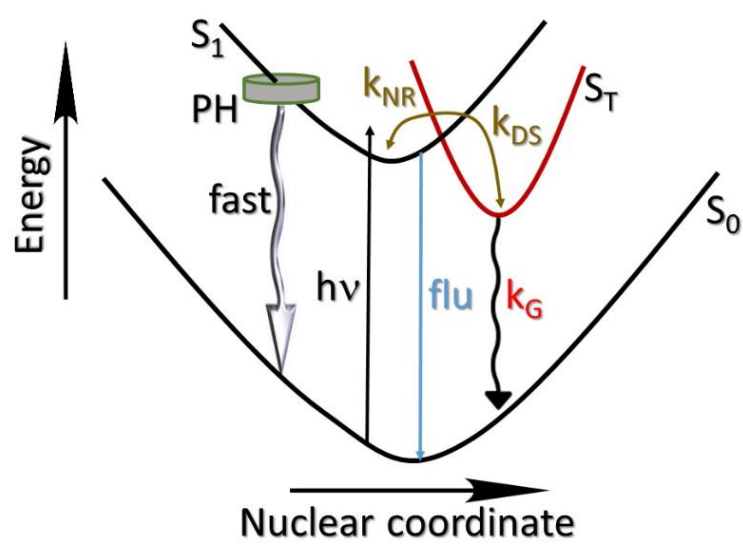

Figure 9. Illustration of the potential energy surfaces proposed for the target compound in a fluid solvent. Fast transformation to the ground state accompanies entry of the pinhole sink $(\mathrm{PH})$, which is populated in competition with access to the conformational trap. The model allows for slow relaxation to the ground state via the trap. Note the alternative arrangement associates the pinhole sink with the conformational trap so that the two nonradiative channels act in tandem.

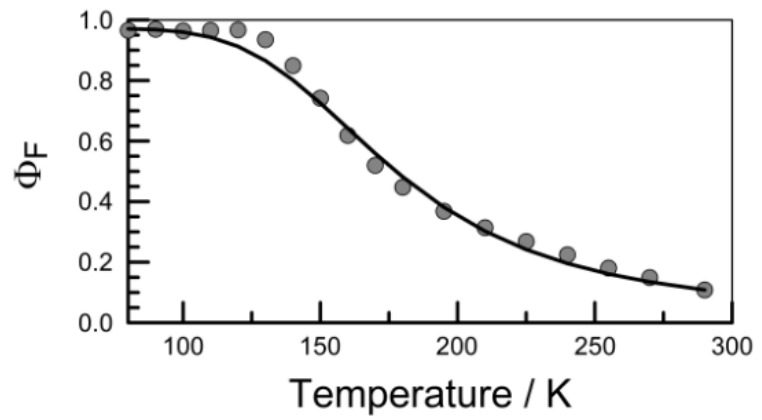

Figure 1o. Effect of temperature on the fluorescence quantum yield recorded for BDFO in MTHF. The solid line drawn through the data points corresponds to a fit to a modified version of Equation 9 where an activated rate constant has been added to account for the rotary action of the meso-tolyl ring.

lifetimes using a model where the pinhole appeared on the $\mathrm{S}_{\mathrm{T}}$ surface (Figure S16). Using reasonable parameters, the temperature dependence for $\Phi_{\mathrm{F}}$ could be reproduced but the same parameters did not allow simulation of the excited-state lifetimes. Simulating the lifetimes resulted in a separate set of parameters, which looked reasonable, but could not reproduce the quantum yields. This dilemma was solved by separating the pinhole sink from the conformational trap (Figure 9). Now, a single set of parameters could be found by iteration that reproduced both lifetimes and quantum yields as a function of temperature. The resultant fit of the $\Phi_{\mathrm{F}}$ values is shown as Figure 10. It might be noted that the fit is far from perfect in the region where the amorphous glass melts to a fluid solution. This indicates that further subtleties involving interactions between 
solvent and solute contribute to the conformational mobility.

The model used to derive the parameters appropriate for fluid solution is provided as part of the Supporting Information. Also given is a collection of the experimental and simulated fluorescence lifetimes obtained using these parameters. The actual parameters are included as part of Table 2, where comparison can be made with the analysis for SOA. The most significant change between SOA and MTHF relates to $k_{N R}$, which is greatly increased in the amorphous sugar matrix. This is surely a consequence of disparate geometries established for the two cases. In fact, $\mathrm{k}_{\mathrm{NR}}$ is important only at lower temperatures for MTHF. The viscosity dependence is associated with accessing the pinhole sink and it is likely that the associated activation energy ${ }^{57} E_{P}$ is sensitive to the viscosity of the solvent.

It should be mentioned that no long-lived (i.e., >10 ns) intermediate species could be detected following pulsed laser excitation of BDFO in fluid solution. In particular, the triplet-excited state was not evident under these experimental conditions. Also significant is the observation that the presence of molecular oxygen had no effect on the dynamics of the various processes discussed above. It might be mentioned here that an earlier report has suggested a link between the circularly polarized luminescence from a doubly-strapped BODIPY analogue and an intramolecular charge-transfer state. ${ }^{5}$ Our spectroscopic studies do not indicate direct involvement of charge-transfer character for BDFO. Rotation around the connection between the dipyrrin unit and the meso-aryl ring does affect the HOMOLUMO energy gap, most notably by perturbing the LUMO (Figures S28 and 29).

Natural transition orbitals ${ }^{59,60}$ have been computed for the first three optical transitions (Figure S30). For the first such transition, essentially HOMO-LUMO, the hole is spread over the strapped aryl ring, including the $\mathrm{O}$ atom, and the attached pyrrole unit. There is no involvement of either the meso- $\mathrm{C}$ atom or the meso-aryl ring. In contrast, the electron is more narrowly distributed over the dipyrrin unit, including the meso- $\mathrm{C}$ atom, with minor contributions from the meso-aryl ring. This situation confers a small dipolar polarization, giving rise to a small but definite intramolecular charge-transfer character. The second transition, nominally $\mathrm{HOMO}(-1)-\mathrm{LUMO}$, shows similar natural transition orbitals (Figure S30), with the hole being located further from the dipyrrin unit and nicely spread over the strapped aryl ring. This situation should augment the charge-transfer character because of increased spatial isolation. The band gap for the second transition, however, is far removed from that for the first transition. Thus, on the basis of charge-transfer excitations ${ }^{61}$ for BDFO, we can expect weak push-pull electronic properties that will be sensitive to the mutual geometry of the respective subunits. It should be stressed that the computational methodology used here is not ideal for resolving charge-transfer character. $^{62}$

\section{CONCLUSIONS}

The mono-strapped BODIPY derivative studied $^{12}$ here displays complex photophysical behavior, especially in solution, in marked contrast to the non-strapped analogue. The strap imposes structural distortion, forcing the dipyrrin unit to adopt a hemispherical dome-shaped structure, and introduces significant asymmetry for both groundand excited-state geometries. An interesting consequence of such distortion is the appearance of E-type delayed fluorescence. ${ }^{63,64}$ Our interpretation of this behavior is that a meta-stable structure is reached at elevated temperatures, at least for the excited-singlet state. Exit from this trapped state can lead to either the ground state, aided by gyration of the meso-aryl ring, or to the emitting singlet-excited state. The interplay between these two avenues is sensitive to the environment but the complexity of the overall system does not make for a viable fluorescent rotor. Measuring viscosity by way of delayed fluorescence might be an interesting concept but requires specialized instrumentation and is fairly slow.

In conventional terms, E-type delayed fluorescence is associated with reversible population of triplet-excited states, ${ }^{65-69}$ excimers, $^{70}$ exciplexes $^{71}$ or intramolecular charge-transfer states. ${ }^{72,73}$ The respective energy gaps between emitter and trap are of critical importance but often can be modulated by conformational changes. ${ }^{74,75}$ Thus, the importance of molecular topology in controlling the yield and lifetime of any delayed fluorescence has already been recognized. ${ }^{6,77}$ Likewise, the fluorescence blinking seen during super-resolution microscopy, and often attributed to reactions between the triplet-excited state and redox-active buffer, ${ }^{78-80}$ could easily be related to light-induced isomerization. ${ }^{81}$ Again, this is a reversible conformational change leading to formation of a meta-stable species. $^{82,83}$ Our understanding of the photophysics of BDFO is that the emitting state is (re-)populated from a geometrically distorted trap that is not a triplet-excited state. It is not possible to rule out the involvement of intramolecular charge-transfer interactions but all indications point to internal structural changes playing an important role.

We do not possess any real information about the nature of the trap proposed for the strapped BODIPY derivatives. It represents a dark state but is raised from obscurity by the observation of thermally-activated, delayed fluorescence from the BODIPY unit. The lifetime for this delayed fluorescence at room temperature is reasonably long, provided access to the pinhole is blocked, but much shorter than that associated with excited-triplet state traps. The pinhole is common to all BODIPY-based molecular rotors and involves concerted translation of both rotor and the upper rim of the dipyrrin unit. More information about the trap might be gleaned from studies made with the corresponding strapped BODIPY dyes that lack the rotor at the mesosite. We should be able to comment on this situation in the near future.

\section{AUTHOR INFORMATION}

\section{Corresponding Author}


*Anthony.harriman@ncl.ac.uk: tel. +44191 208866o. ORCID oooo-0003-0679-2232.

\section{Author Contributions}

All authors have given approval to the final version of the manuscript.

\section{ACKNOWLEDGMENT}

We thank Newcastle University and the Leverhulme Trust for financial support of this work.

\section{ABBREVIATIONS}

MTHF, 2-methyltetrahydrofuran; SOA, sucrose octaacetate; BODIPY, boron dipyrromethene dye.

\section{ASSOCIATED CONTENT}

Supporting Information. Provided are experimental details, examples and procedures for Gaussian analysis of spectra, details for solvent and temperature effects, model for decay in fluid solution, computational details and temperature dependence for MTHF properties. This material is available free of charge via the Internet at http://pubs.acs.org.

\section{REFERENCES}

(1) Haidekker, M. A.; Theodorakis, E. A.; Molecular Rotors Fluorescent Biosensors for Viscosity and Flow. Org. Biomol. Chem. 2007, 5, 1669-1678.

(2) Sasaki, S.; Drummon, G. P. C.; Konishi, G. Recent Advances in Twisted Intramolecular Charge Transfer (TICT) Fluorescence and Related Phenomena in Materials Chemistry. J. Mater. Chem. C 2016, 4, 2731-2743.

(3) Hawe, A.; Sutter, M.; Jiskoot, W. Extrinsic Fluorescent Dyes as Tools for Protein Characterization. Pharm. Res. 2008, 25, 14871499.

(4) Peng, X. J.; Yang, Z. G.; Wang, J. Y.; Han, J. L.; He, Y. X.; Song, F. L.; Wang, B. S.; Sun, S. G.; Qu, J. L.; Qi, J.; Yang, M. Fluorescence Ratiometry and Fluorescence Lifetime Imaging. Using a Single Molecular Sensor for Dual Mode Imaging of Cellular Viscosity. J. Am. Chem. Soc. 2011, 133, 6626-6635.

(5) Levitt, J. A.; Chung, P. H.; Kuimova, M. K.; Yahioghu, G.; Wang, Y.; Qu, J. L.; Suhling, K. Fluorescence Anisotropy of Molecular Rotors. ChemPhysChem 2011, 12, 662-672.

(6) Giggino, K. P.; Hutchison, J. A.; Langford, S. J.; Latter, M.; Lee, M. A. P.; Lowenstern, P. R.; Scholes, C.; Takezaki, M.; Wilman, B. E. Porphyrin-Based Molecular Rotors as Fluorescent Probes of Nanoscale Environments. Adv. Funct. Mater. 2007, 17, $805-813$.

(7) Boens, N.; Leen, V.; Dehaen, W. Fluorescent Indicators Based on BODIPY. Chem. Soc. Rev. 2012, 41, 1130-1172.

(8) Levitt, J. A.; Kuimova, M. K.; Yahioghu, G.; Chung, P. H.; Suhling, K.; Phillips, D. Membrane-Bound Molecular Rotors Measure Viscosity in Live Cells via Fluorescence Lifetime Imaging. J. Phys. Chem. C 2009, 113, 11634-11642.

(9) Alamiry, M. A. H.; Benniston, A. C.; Copley, G. C.; Elliott, K. J.; Harriman, A.; Stewart, B.; Zhi, Y. G. A Molecular Rotor Based on an Unhindered Boron Dipyrromethene (Bodipy) Dye. Chem. Mater. 2008, 20, 4024-4032.

(10) Ulrich, G.; Ziessel, R.; Harriman, A. The Chemistry of Fluorescent Bodipy Dyes: Versatility Unsurpassed. Angew. Chem., Int. Ed. 2008, 47, 1184-1201.

(11) Bahaidarah, E.; Harriman, A.; Stachelek, P.; Rihn, S.; Heyer, E.; Ziessel, R. Fluorescent Molecular Rotors Based on the BODIPY Motif: Effect of Remote Substituents. Photochem. Photobiol. Sci. 2014, 13, 1397-1401.
(12) Sirbu, D.; Benniston, A. C.; Harriman, A. One-Pot Synthesis of a Mono-O,B,N-Strapped BODIPY Derivative Displaying Bright Fluorescence in the Solid State. Org. Lett. 2017, 19, 1626-1629.

(13) Ziessel, R.; Ulrich, G,; Harriman, A. The Chemistry of Bodipy: A New El Dorado for Fluorescence Tools. New J. Chem. 2007, 31, 496-501.

(14) Loudet, A.; Burgess, K. BODIPY Dyes and Their Derivatives. Synthesis and Spectroscopic Properties. Chem. Rev. 2007, 107, 4891-4932.

(15) Qin, W. W.; Baruah, M.; Van der Auweraer, M.; De Schryver, F. C.; Boens, N. Photophysical Properties of Boron Dipyrromethene Analogues in Solution. J. Phys. Chem. A 2005, 109, 7371-7384.

(16) Burghart, A.; Kim, H. J.; Welch, M. B.; Thoresen, L. H.; Reibenspies, J. Burgess, K.; Bergstrom, F.; Johansson, L. B. A. 3,5Diaryl-4,4-difluoro-4-bora-3a,4a-diaza-s-indacene (BODIPY) Dyes: Synthesis, Spectroscopic, Electrochemical, and Structural Properties. J. Org. Chem. 1999, 64, 7813-7819.

(17) Nepomnyashchii, A. B.; Bröring, M.; Ahrens, J.; Bard, A. J. Chemical and Electrochemical Dimerization of BODIPY Compounds. Electrogenerated Chemiluminescent Detection of Dimer Formation. J. Am. Chem. Soc. 2011, 133, 19498-19504.

(18) Milian-Medina, B.; Gierschner, J. Computational Design of Low Singlet-Triplet Gap All-Organic Molecules for OLED Application. Org. Electron. 2013, 13, 985-991.

(19) Sun, H. T.; Zhong, C.; Brédas, J. L. Reliable Prediction with Tuned Range-Separated Functionals of the Singlet-Triplet Gap in Organic Emitters for Thermally-Activated Delayed Fluorescence. J. Chem. Theory Comput. 2015, 11, 3851-3858.

(20) O'Connor, D. V.; Phillips, D. Time-Correlated Single Photon Counting. Academic Press, London, 1984.

(21) Van Den Zegel, M.; Boens, N.; De Schryver, F. C. Possibilities and Limitations of the Time-Correlated Single Photon Counting Technique: A Comparative Study of Correction Methods for the Wavelength Dependence of the Instrument Response Function. Chem. Phys. 1986, 101, 311-335.

(22) Heitele, H.; Finckh, P.; Weeren, S.; Pöllinger, F.; MichelBeyerle, M. E. Solvent Polarity Effects on Intramolecular Electron Transfer. 1. Energetic Aspects. J. Phys. Chem. 1989, 93, 5173-5179.

(23) Velsko, S. P.; Fleming, G. R. Photochemical Isomerization in Solution - Photophysics of Diphenyl Butadiene. J. Chem. Phys. 1982, 76, 3553-3562.

(24) Fleming, G. R.; Courtney, S. H.; Balk, M. W. Activated Barrier Crossing - Comparison of Experiment and Theory. J. Stat. Phys. 1986, 42, 83-104.

(25) Alamiry, M. A. H.; Bahaidarah, E.; Harriman, A.; Bura, T.; Ziessel, R. Fluorescent Molecular Rotors under Pressure: Synergistic Effects of an Inert Polymer. RSC Adv. 2012, 2, 9851-9859.

(26) Sun, Y. P.; Saltiel, J.; Hoburg, E. A.; Waldeck, D. H. Application of the Medium-Enhanced Model to the Photoisomerization Dynamics of Substituted Stilbenes in Normal Alkane Solvents. J. Phys. Chem. 1991, 95, 10336-10344.

(27) Förster, T.; Hoffmann, G. Die Viskositätsabhängigkeit der Fluoreszenzquantenausbeuten Einiger Fabstroffsysteme. Zeit. Physik. Chem. NF 1971, 75, 377-413.

(28) Akesson, E.; Hakkarainen, A.; Laitinen, E.; Helenius, V.; Gillbro, T. Analysis of Microviscosity and Reaction Coordinate Concepts in Isomerization Dynamics Described by Kramers Theory. J. Chem. Phys. 1991, 95, 6508-6523.

(29) Zimdars, D.; Tokmakoff, A.; Chen, S.; Greenfield, S. R.; Fayer, M. D. Picosecond Infrared Vibrational Photon Echoes in a Liquid and Glass Using a Free Electron Laser. Phys. Rev. Lett. 1993 70, 125-128

(30) Görlach, E.; Gygax, H.; Lubini, P.; Wild, U. P. Solvent Relaxation of Oxazine-4 in 2-Methyltetrahydrofuran. Chem. Phys. 1995, 194, 185-193. 
(31) Engler, B. P.; Harrah, L. A. Viscosity and Density of 2-Methyltetrahydrofuran as a Function of Temperature. SAND - 781414. 1979.

(32) Al-Aqar, R.; Benniston, A. C.; Harriman, A.; Perks, T. Structural Dynamics and Barrier Crossing Observed for a Fluorescent O-Doped Polycyclic Aromatic Hydrocarbon. ChemPhotoChem 2017, 1, 198-205.

(33) Kaposi, A. D.; Wright, W. W.; Vanderkooi, J. M. Consequences of Inhomogeneous Broadening on Fluorescence Line Narrowing Spectra. J. Fluoresc. 2003, 13, 59-67.

(34) Paulheim, A.; Marquardt, C.; Aldahhak, H.; Rauls, E.; Schmidt, W. G.; Sokolowski, M. Inhomogeneous and Homogeneous Line Broadening of Optical Spectra of PTCDA Molecules Adsorbed at Step Edges of Alkali Halide Surfaces. J. Phys. Chem. C 2016, 120, 11926-11937.

(35) Nemkovich, N. A.; Rubinov, A. N.; Tomin, V. I. Inhomogeneous Broadening of Electronic Spectra of Dye Molecules in Solution. In Topics in Fluorescence Spectroscopy. Ed. Lakowitz, J. Plenum Press, New York, 1991.

(36) Stein, A. D.; Fayer, M. D. Nanosecond Timescale Optical Inhomogeneous Broadening of Dye Molecules in Liquids at and Near Room Temperature. Chem. Phys. Lett. 1991, 176, 159-166.

(37) Peterman, E. J. G.; Pullerits, T.; van Grondelle, R. ElectronPhonon Coupling and Vibronic Fine Structure of Light-Harvesting Complex II of Green Plants: Temperature Dependent Absorption and High-Resolution Fluorescence Spectroscopy. J. Phys. Chem. B 1997, 101, 4448-4457.

(38) Roos, C.; Kohn, A.; Gauss, J.; Diezemann, G. The Temperature Dependence of Vibronic Lineshapes: Linear Electron-Phonon Coupling. J. Chem. Phys. 2014, 154110.

(39) Sevian, H. M.; Skinner, J. L. A Molecular Theory of Inhomogeneous Broadening, Including the Correlation Between Different Transitions, in Liquids and Glasses. Theor. Chim. Acta 1992, $82,29-46$.

(40) Lee, H. W. H.; Walsh, C. A.; Fayer, M. D. Inhomogeneous Broadening of Electronic Transitions of Chromophores in Crystals and Glasses: Analysis of Hole Burning and Fluorescence Line Narrowing Experiments. J. Chem. Phys. 1985, 82, 3948-3958.

(41) Karolin, J.; Johannsen, L. B.-Å.; Strandberg, L.; Ny, T. Fluorescence and Absorption Spectroscopic Properties of Dipyrromethene Difluoride (BODIPY) derivatives in Liquids, Lipid Membranes, and Proteins. J. Am. Chem. Soc. 1994, 116, 7801-7806.

(42) Vacha, M.; Liu, Y.; Nakatsuka, H.; Tani, T. Inhomogeneous and Single Molecule Line Broadening of Terrylene in a Series of Crystalline n-Alkanes. J. Chem. Phys. 1997, 106, 8324-8331.

(43) Tao, Y.; Yuan, K.; Chen, T.; Xu, P.; Li, H. H.; Chen, R. F.; Zheng, C.; Zhang, L.; Huang, W. Thermally Activated Delayed Fluorescence Materials Towards the Breakthrough of Organoelectronics. Adv. Mater. 2014, 26, 7931-7958.

(44) Zhang, Q. S.; Li, B.; Huang, S. P.; Nomura, H.; Tanaka, H.; Adachi, C. Efficient Blue Organic Light-Emitting Diodes Employing Thermally Activated Delayed Fluorescence. Nat. Photonics 2014, 8, 326-332.

(45) Mehes, G.; Nomura, H.; Zhang, Q. S.; Nahagawa, T.; Adachi, C. Enhanced Electroluminescence Efficiency in a SpiroAcridine Derivative Through Thermally Activated Delayed Fluorescence. Angew. Chem., Int. Ed. 2012, 51, 11311-11315.

(46) Baleizão, C.; Berberan-Santos, M. N. Thermally Activated Delayed Fluorescence in Fullerenes. Ann. New York Acad. Sci. 2008, 1130, 224-234.

(47) Rothe, C.; King, S. M.; Dias, F.; Monkman, A. P. Triplet Exciton State and Related Phenomena in the beta-Phase of Poly(9,9dioctyl)fluorene. Phys. Rev. 2004, 70, 195213.

(48) Penfold, T. J. On Predicting the Excited-State Properties of Thermally Activated Delayed Fluorescence Emitters. J. Phys. Chem. C 2015, 119, 13535-13544.
(49) Stachelek, P.; Alsimaree, A. A.; Alnoman, R. B.; Harriman, A.; Knight, J. G. Thermally-Activated, Delayed Fluorescence in $\mathrm{O}, \mathrm{B}, \mathrm{O}-$ and N,B,O-Strapped Boron Dipyrromethene Derivatives. J. Phys. Chem. A 2017, 121, 2096-2107.

(50) Baleizão, C.; Berberan-Santos, M. N. Thermally Activated Delayed Fluorescence as a Cycling Process Between Excited Singlet and Triplet States: Application to the Fullerenes. J. Chem. Phys. 2007, 126, 204510.

(51) Dias, F. B.; Penfold, T. J.; Monkman, A. P. Photophysics of Thermally Activated Delayed Fluorescence Molecules. Methods Appl. Fluoresc. 2017, 5, 012001.

(52) Palmeira, T.; Berberan-Santos, M. N. Kinetic Criteria for Optimal Thermally-Activated Delayed Fluorescence in Photoluminescence and in Electroluminescence. J. Phys. Chem. C 2017, 121, 701-708.

(53) Strickler, S. J.; Berg, R. A. Relation between Absorption Intensity and Fluorescence Lifetime of Molecules. J. Chem. Phys. 1962, 37, 814-822.

(54) Kee, H. L.; Kirmaier, C.; Yu, L. H.; Thamyongkit, P.; Youngblood, W. J.; Calder, M. E.; Ramos, L.; Noll, B. C.; Bocian, D. F.; Scheidt, W. R.; Birge, R. R.; Lindsey, J. S.; Holten, D. Structural Control of the Photodynamics of Boron Dipyrrin Complexes. J. Phys. Chem. B 2005, 109, 20433-20443.

(55) Bagchi, B.; Fleming, G. R.; Oxtoby, D. W. Theory of Electronic Relaxation in Solution in the Absence of an Activation Barrier. J. Chem. Phys. 1983, 78, 7375-7385.

(56) Rucker, R. L.; Schwartz, B. J.; El-Bayoumi, M. A.; Harris, C. B. Ultrafast Dynamics of Sterically Hindered Tetraphenylmethylbutadiene in Liquids. Chem. Phys. Lett. 1995, 235, 471-478.

(57) Alnoman, R. B.; Rihn, S.; O'Connor, D. C.; Black, F. A.; Costello, B.; Waddell, P. G.; Clegg, W.; Peacock, R. D.; Herrebout, W.; Knight, J. G.; et al. Circularly Polarized Luminescence from Helically Chiral N,N,O,O-Boron Chelated Dipyrromethenes. Chem. Eur. J. 2016, 22, 93-96.

(58) Clarke, R.; Ho, K. L.; Alsimaree, A. A.; Woodford, O. J.; Waddell, P. G.; Bogaerts, J.; Herrebout, W.; Knight, J. G.; Pal, R.; Penfold, T. J.; Hall, M. J. Circularly Polarised Luminescence from Helically Chiral "Confused" N,N,O,C-Boron-Chelated Dipyrromethenes (BODIPYs). ChemPhotoChem 2017, 1, 513-517.

(59) Martin, R. L. Natural Transition Orbitals. J. Chem. Phys. 2003, 118, 4775-4777.

(6o) Xochitiotzi, E.; Jimenez-Sanchez, A.; Garcia-Ortega, H.; Sanchez-Puig, N.; Romero-Avila, M.; Santillan, R.; Farfan, N. Optical Properties of Two Fluorene-Derived BODIPY Molecular Rotors as Fluorescent Ratiometric Viscosity Probes. New J. Chem. 2016, 40, 4500-4512.

(61) Le Bahers, T.; Adamo, C.; Ciofini, I. A Qualitative Index of Spatial Extent in Charge-Transfer Excitations. J. Chem. Theory Comput. 2011, 7, 2498-2506.

(62) Veldkamp, B. S.; Liu, X.; Wasielewski, M. R.; Subotnik, J. E.; Ratner, M. A. Molecular Excited States: Accurate Calculation of Relative Energies and Electronic Coupling Between Charge Transfer and Non-Charge Transfer States. J. Phys. Chem. A 2015, 119, 253-262.

(63) Razinaqvi, K.; Wild, U. P. Use of E-Type Delayed Fluorescence for Probing Rotational Relaxation. Chem. Phys. Lett. 1975 36, 222-224.

(64) Jankus, V.; Chiang, C. J.; Dias, F.; Monkman, A. P. Deep Blue Exciplex Organic Light-Emitting Diodes with Enhanced Efficiency; P-Type and E-Type Triplet Conversion to Singlet Excitons? Adv. Mater. 2013, 25, 1455-1459.

(65) Okazaki, M.; Takeda, Y.; Data, P.; Pander, P.; Higginbotham, H.; Monkman, A. P.; Minakata, S. Thermally-Activated Delayed Fluorescent Phenothiazine-Dibenzo[a,j]phenazine-Phenothiazine Triads Exhibiting Tricolor-Changing Mechanochromic Luminescence. Chem. Sci. 2017, 8, 2677-2686. 
(66) Olivier, Y,; Yurash, B.; Muccioli, L.; D’Avino, G,; Mikhnenko, O.; Sancho-Garcia, J. C.; Adachi, C.; Nguyen, T.-Q.; Beljonne, D. Nature of the Singlet and Triplet Excitations Mediating Thermally-Activated Delayed Fluorescence. Phys. Rev. Mater. 2017, 1, 075602.

(67) Cui, X. N.; El-Zohry, A. M.; Wang, Z. J.; Zhao, J. Z.; Mohammed, O. F. Homo- or Hetero-Triplet Triplet Annihilation? A Case Study with Perylene-BODIPY Dyads/Triads. J. Phys. Chem. C 2017, 121, 16182-16192.

(68) Ishimatsu, R.; Edura, T.; Adachi, C.; Nakano, K.; Imato, T. Photophysical Properties and Efficient, Stable, Electrogenerated Chemiluminescence of Donor-Acceptor Molecules Exhibiting Thermal Spin Conversion. Chem. Eur. J. 2016, 22, 4889-4898.

(69) Fan, J. Z.; Zhang, Y. C.; Zhou, Y.; Lin, L. L.; Wang, C. K. Excited State Properties of a Thermally Activated Delayed Fluorescence Molecule in Solid Phase Studied by Quantum Mechanics/Molecular Mechanics Method. J. Phys. Chem. C 2018, 122, 23582366.

(7o) Alamiry, M. A. H.; Benniston, A. C.; Copley, G.; Harriman, A.; Howgego, D. Intramolecular Excimer Formation for Covalently Linked Boron Dipyrromethene Dyes. J. Phys. Chem. A 2011, 115, 12111-12119.

(71) Cao, H. T.; Zhao, Y.; Sun, C.; Fang, D.; Xie, L. H.; Yan, M. N.; Wei, Y.; Zhang, H. M.; Huang, W. Novel Electron Acceptor Based on Spiro [fluorene-9,9'-xanthene] for Exciplex Thermally Activated Delayed Fluorescence. Dyes Pigm. 2018, 149, 422-429.

(72) Kuang, Z. R.; He, G. Y.; Song, H. W.; Wang, X.; Hu, Z. B.; Sun, H. T.; Wan, Y.; Guo, Q. J.; Xia, A. D. Conformational Relaxation and Thermally Activated Delayed Fluorescence in Anthraquinone-Based Intramolecular Charge-Transfer Compound. J. Phys. Chem. C 2018, 122, 3727-3737.

(73) Kim, K. J.; Kim, G. H.; Lampande, R.; Ahn, D. H.; Im, J. B.; Moon, J. S.; Lee, J. K.; Lee, J. Y.; Kwon, J. H. A New Rigid-Diindolocarbazole Donor Moiety for High Quantum Efficiency Thermally Activated Delayed Fluorescence Emitter. J. Mater. Chem. C 2018, 6, 1343-1348.

(74) Choi, J.; Ahn, D. S.; Oang, K. Y.; Cho, D. W.; Ihee, H. Charge Transfer-Induced Torsional Dynamics in the Excited State of 2,6-Bis(diphenylamino)anthraquinone. J. Phys. Chem. C 2017, 121, 24317-24323.

(75) Joshi, R.; Meitei, O. R.; Jadhao, M.; Kumar, H.; Ghosh, S. K. Conformation Controlled Turn On Turn Off Phosphorescence in a Metal-Free Biluminophore: Thriving the Paradox that Exists for Organic Compounds. Phys. Chem. Chem. Phys. 2016, 18, 2791027920.

(76) Aydemir, M.; Haykir, G.; Turksoy, F.; Gumus, S.; Dias, F. B.; Monkman, A. P. Synthesis and Investigation of Intramolecular Charge Transfer State Properties of Novel Donor-Acceptor-Donor Pyridine Derivatives: The Effects of Temperature and Environment on Molecular Configurations and the Origin of Delayed Fluorescence. Phys. Chem. Chem. Phys. 2015, 17, 25572-25582.

(77) Cazeau-Dubroca, C.; Peirigua, A.; Lyazidi, S. A.; Nouchi, G.; Cazeau, P.; Lapouyade, R. TICT Fluorescence in Rigid Matrices $\alpha$-Delayed Fluorescence. Chem. Phys. Lett. 1986, 124, 110-115.

(78) Galievsky, V. A.; Victor, A.; Druzhinin, S. I.; Demeter, A.; Mayer, P.; Kovalenko, S. A.; Senyushkina, T. A.; Zachariasse, K. A. Ultrafast Intramolecular Charge Transfer with N-(4-Cyanophenyl)carbazole. Evidence for a LE Precursor and Dual LE Plus ICT Fluorescence. J. Phys. Chem. A 2010, 114, 12622-12638.

(79) Dave, R.; Terry, D. S.; Munro, J. B.; Blanchard, S. C. Mitigating Unwanted Photophysical Processes for Improving SingleMolecule Fluorescence Imaging. Biophys. J. 2009, 96, 2371-2381.

(8o) van der Velde, J. H. M.; Ploetz, E.; Hiermaier, M.; Oelerich, J.; de Vries, J. W.; Roelfes, G.; Cordes, T. Mechanism of Intramolecular Photostabilization in Self-Healing Cyanine Fluorophores. ChemPhysChem 2013, 14, 4084-4093.

(81) Ciuba, M. A.; Levitus, M. Manganese-Induced Triplet Blinking and Photobleaching of Single Molecule Cyanine Dyes. ChemPhysChem 2013, 14, 3495-3502.

(82) Levitus, M.; Ranjit, S. Cyanine Dyes in Biophysical Research: The Photophysics of Polymethine Fluorescent Dyes in Biomolecular Environments. Quart. Rev. Biophys. 2011, 44, 123-151.

(83) Stennett, E. M. S.; Ciuba, M. A.; Levitus, M. Photophysical Processes in Single Molecule Organic Fluorescent Probes. Chem. Soc. Rev. 2014, 43, 1057-1075. 
Table of Contents artwork

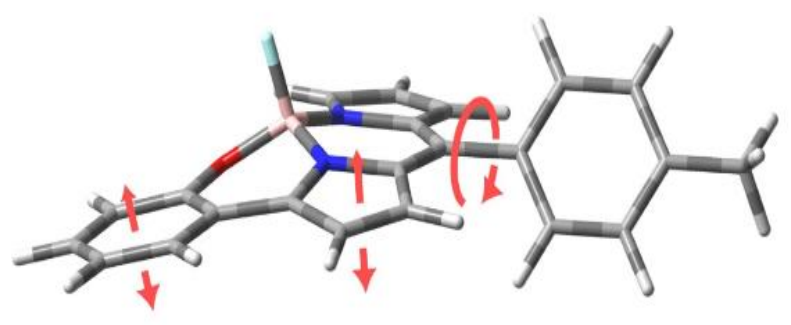

